\title{
DESCRIPCIÓN DE PARÁSITOS PRESENTES EN MUESTRAS FECALES RECOLECTADAS EN PLAZAS DEL SECTOR CÉNTRICO DE LA CIUDAD DE TEMUCO, CHILE
}

\author{
Description of Parasites in Faecal Samples Collected in Plazas of \\ Downtown Temuco, Chile
}

Pamela Olivares ${ }^{1,2}$, Gastón Valenzuela ${ }^{2}$, Christian Tuemmers ${ }^{2}$, Jorge Parodi ${ }^{1,3}$

\section{Resumen}

El objetivo del estudio fue determinar la contaminación de plazas públicas de la ciudad de Temuco con formas parasitarias a través de las heces de perros. Se recolectaron 102 muestras de materia fecal de ocho plazas del centro de la ciudad. Las muestras fueron procesadas por el método de flotación-sedimentación (método de Teuscher). El $89.2 \%$ de las muestras dieron positivo para alguna forma parasitaria, donde los huevos tipo estrongílido (69.6\%) dentro del Phylum Nematoda y los ooquistes de Isospora dentro del Phylum Protozoa (6.8\%) fueron los más frecuentes. El 50\% de las muestras fueron poliparasitarias, destacando el triparasitismo, que alcanzó el $21.5 \%$ del total de muestras analizadas. Los hallazgos indican un ambiente contaminado y un mal manejo de residuos en el centro de la ciudad.

Palabras clave: contaminación de parques, estrongílidos, poliparasitismo, perros

\section{AbSTRACT}

The aim of this study was to determine the level of contamination of public plazas in the city of Temuco with parasitic forms through dog faeces. A total of 102 faecal samples were collected in eight plazas of the city centre. Samples were processed by the sedimentation-flotation method (Teuscher method). The $89.2 \%$ of the samples resulted

${ }^{1}$ Laboratorio de Fisiología de la Reproducción, Escuela de Medicina Veterinaria, Núcleo de Investigación en Producción Alimentaria, Facultad de Recursos Naturales, Universidad Católica de Temuco, Teтuco, Chile

${ }^{2}$ Escuela de Medicina Veterinaria, Facultad de Recursos Naturales, Universidad Católica de Temuco, Temuco, Chile

${ }^{3}$ E-mail:jparodi@uct.cl

Recibido: 3 de enero de 2014

Aceptado para publicación: 17 de marzo de 2014 
positive for a parasitic form, where strongyle-type eggs (69.6\%) within the Phylum Nematoda and the oocysts of Isospora within the Phylum Protozoa (6.8\%) were the most frequent. Half of the samples were poliparasitic where the triparasitism reached $21.5 \%$ of all samples. The findings indicate a polluted environment and poor waste management in the city center.

Key words: contamination of public places, strongylus, poliparasitism, dogs

\section{INTRODUCCIÓN}

El hombre es un hospedero habitual de parásitos internos y externos, condición que merece especial atención debido a la estrecha relación que existe entre las personas y sus mascotas (Boch y Supperer, 1982). El perro doméstico (Canis familiaris) es el principal agente involucrado en la contaminación de parques y plazas públicas (Armstrong et $a l ., 2011)$. Más aún, estos espacios no solo son vías de tránsito para las personas sino que son lugares donde juegan los niños, siendo la población infantil uno de los grupos más expuestos a los focos de transmisión (Milano y Oscherov, 2005).

Las áreas verdes de parques y plazas públicas son las que representan «áreas de riesgo» o áreas donde las personas tienen un mayor contacto con elementos parasitarios presentes en la materia fecal de perros vagos o perros cuyos propietarios los llevan para que defequen en esos lugares (Salinas et al., 1987; Noemí y Rugiero 1998). El elevado número de perros vagabundos, así como la ausencia de una tenencia responsable de mascotas y de una legislación adecuada en la eliminación de excretas en lugares públicos son causantes de la contaminación de estas áreas verdes (López, 2006).

Entre las especies parasitarias de carácter zoonótico se señala a Toxocara canis como implicado en el Síndrome de larva migrans visceral (LMV) y en el Síndrome de larva migrans ocular (LMO). Este parásito pertenece a la familia Ascaridae y al género
Toxocara y se localiza en el intestino delgado del perro y el zorro (Soulsby, 1965). Las infecciones intestinales con pocos parásitos suelen ser asintomáticas en los animales adultos (Acha y Szyfres, 2003).

Las hembras de $T$. canis son muy prolíficas, pudiendo liberar hasta 200000 huevos por día, los cuales resisten las condiciones del medio ambiente y muchos desinfectantes de uso común (Diez-Baños et al., 1999). La prevalencia de T. canis en los perros es muy alta, debido sobre todo a la eficacia de la transmisión parenteral, por lo que la mayoría de los cachorros recién nacidos tendrán $T$. canis; no obstante, los perros mayores de seis meses suelen tener menos parásitos adultos en el intestino que los cachorros (Diez-Baños et al., 1999). A pesar de que se ha relacionado una resistencia al parásito con la edad de los perros, se tiene constancia de que estos no desarrollan inmunidad protectora y que pueden contribuir de modo significativo a la contaminación del medio con los huevos del parásito (Diez-Baños et al., 1999). Los huevos presentan en su interior una célula única a partir de la cual se desarrolla una larva en 10 a 15 días, pudiendo mantenerse infectante por 7 a 12 años (Glickman y Schanz, 1981).

En el hombre, el reservorio de Toxocara spp son los perros infectados, siendo la fuente principal de infección el suelo contaminado con huevos infectantes. El mecanismo de transmisión es por ingestión de estos huevos a través de los alimentos, agua o manos contaminadas (Acha y Szyfres, 2003). La revisión bibliográfica sugiere que las principales 
Cuadro 1. Número y procedencia de muestras fecales de perros en parques y plazas de Temuco (2012)

\begin{tabular}{lc}
\hline Ubicación & $\mathrm{N}^{\circ}$ \\
\hline (A) Plaza de Armas Aníbal Pinto & 14 \\
(B) Plaza Teodoro Schmidt & 12 \\
(C) Plaza Dagoberto Godoy & 13 \\
(D) Plaza Recabarren & 13 \\
(E) Feria Libre y Av. Aníbal & 13 \\
$\quad$ Pinto & 12 \\
(F) Av. General Prieto Norte & 12 \\
(G) Av. Balmaceda & 13 \\
(H) Plaza Alonso de Ercilla & 102 \\
\hline \multicolumn{2}{l}{ Total }
\end{tabular}

plazas públicas de la ciudad de Temuco, Chile, presentan un alto grado de contaminación con elementos de diseminación parasitaria, especialmente del tipo estrongílido, presentes en la materia fecal de perro. El presente estudio pretende determinar el nivel de contaminación ambiental con formas parasitarias (protozoos, nematodos y cestodos) en heces de perros en plazas públicas de Temuco, así como identificar las formas parasitarias presentes en las heces.

\section{Materiales y Métodos}

\section{Área de Estudio}

El estudio se realizó en la ciudad de Temuco, ubicada a 675 kilómetros al sur de Santiago de Chile, en la Región de La Araucanía. Se obtuvo una lista de todas las plazas públicas de la zona centro de la ciudad $(n=8)$. Se consideró como área de riesgo toda área verde de las plazas o parques que estuviera expuesta al contacto directo de personas con la materia fecal de los perros (Armstrong et al., 2011).

\section{Material Biológico y Muestreo}

Se recolectaron 102 muestras fecales de perro entre abril y mayo de 2012 . Solo se consideraron heces frescas no deshidratadas en las áreas de riesgo, obviando muestras que se encontraran en calzadas o paseos peatonales. La procedencia de las muestras se presenta en el Cuadro 1.

\section{Obtención y Análisis de Muestras}

Se utilizaron paletas descartables de madera para recolectar aproximadamente 5 $\mathrm{g}$ de material fecal por muestra. Las muestras, en bolsas plásticas, se trasladaron al laboratorio bajo refrigeración, siendo analizadas entre 12 a 24 horas después de la recolección.

Para el análisis de las muestras se usó el método de Teuscher (Teuscher, 1965), que es una derivación del método de Faust (Faust, 1938). Es un método sensible y práctico de demostración de huevos y larvas de parásitos, utilizado para exámenes rutinarios de heces de rumiantes y otras especies. Este método ha demostrado tener una mayor eficiencia en el diagnóstico de huevos de nematodos y cestodos, así como de ooquistes de protozoos (Aguirre, 2006); sin embargo, no se recomienda con fines de investigación donde se deben obtener recuentos precisos de huevos.

\section{Análisis Estadístico}

A menos que sea indicado, los resultados, incluido los análisis de imágenes, son presentados como el promedio \pm SEM. Se aplicó la prueba de «t» Student para determinar diferencias estadísticas entre medias. Un nivel de probabilidad menor a 0.05 fue consi- 
derado estadísticamente significativo. Todos los datos fueron analizados con el programa estadístico Prism 4.0.

\section{Resultados}

Las muestras presentaron gran cantidad de parásitos, con presencia de parásitos de diversas especies (Fig. 1). El $11.0 \pm 4.5 \%$ $(n=10)$ de las muestras resultó negativa a la presencia de parásitos gastrointestinales, en tanto que el $40.0 \pm 6.9$ y $50.0 \pm 6.4 \%$ presentaron mono y poliparasitismo, respectivamente (Fig. 1A).
Los huevos de las formas parasitarias fueron agrupadas en base al género como huevos de Toxocara, Trichuris, Capillaria, Strongyloides y de tipo Strongylus o estrongílido, en tanto que a los protozoarios se les agrupó como ooquistes de Eimeria, de Isospora y de Sarcocystis (Fig. 1B, 1C). No se encontraron huevos de cestodos.

Los huevos de especies de nematodos parásitos más frecuentes fueron los de tipo estrongílido, en tanto que los huevos de Toxocara spp se encontraron en el $21.5 \pm$ $7.0 \%$ de las muestras (Fig. 1B). El número de muestras con presencia de protozoarios
A.

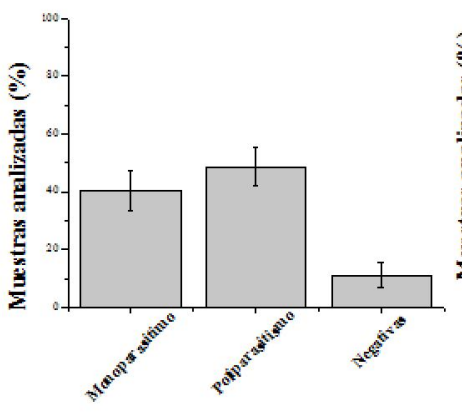

D.
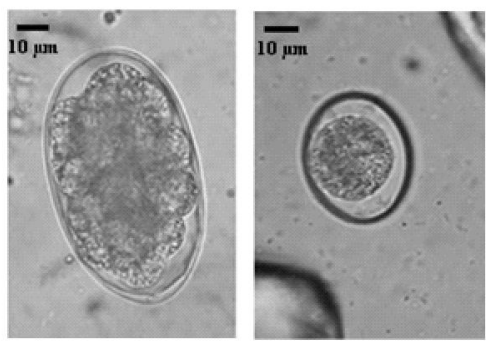

B.

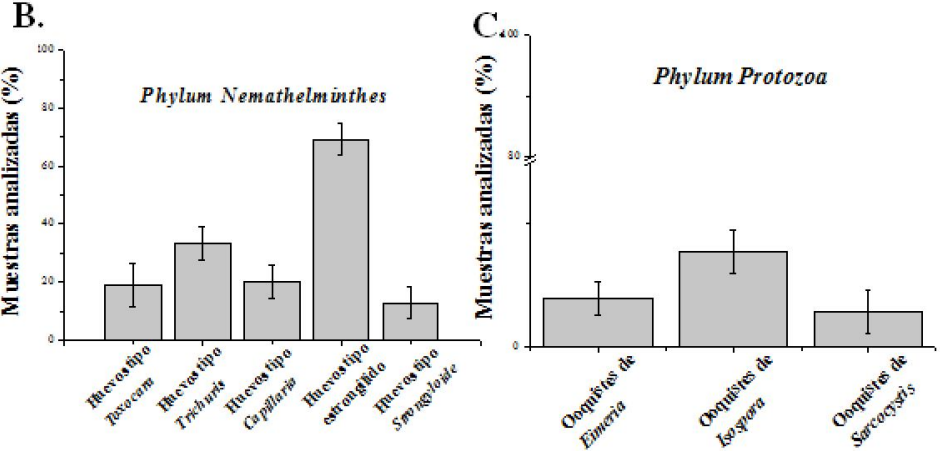

E.

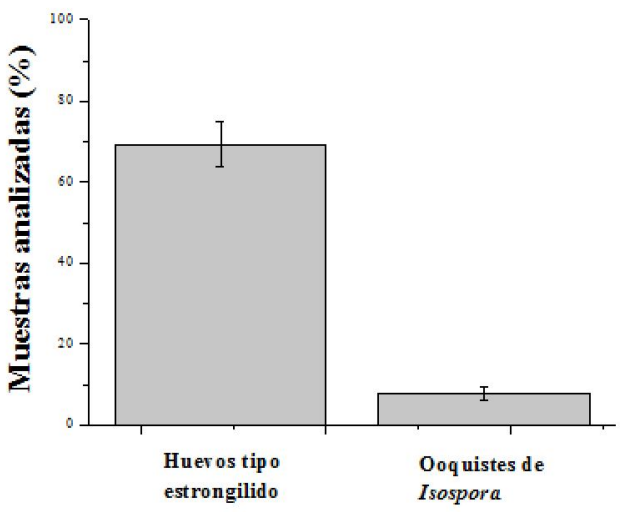

Figura 1. Análisis de muestras de heces de perros recolectadas en plazas públicas en la ciudad de Temuco, Chile (2012). A. Resultados parasitológicos de las muestras analizadas; B. Distribución porcentual de huevos de nematodos; C. Distribución porcentual de ooquistes; D. Microfotografías de huevos tipo strongílido y ooquistes de Isospora spp; E. Frecuencia de huevos tipo estrongílido y de ooquistes en las muestras positivas. Las barras en A, B, C y E indican error estándar 
Cuadro 2. Frecuencia (\%) de huevos de parásitos gastrointestinales hallados en muestras fecales de perros en ocho plazas públicas en la ciudad de Temuco, Chile (2012)

\begin{tabular}{|c|c|c|c|c|c|}
\hline Lugar $^{1}$ & $\begin{array}{l}\text { Huevos tipo } \\
\text { Strongylus }\end{array}$ & Toxocara & Capillaria & Trichuris & Strogyloides \\
\hline A & 85.7 & 71.4 & & & \\
\hline B & 58.3 & & 41.7 & & \\
\hline $\mathrm{C}$ & 92.3 & 15.4 & & & \\
\hline $\mathrm{D}$ & 38.5 & & & 53.8 & \\
\hline $\mathrm{E}$ & 69.2 & & & 61.5 & \\
\hline $\mathrm{F}$ & 58.3 & & & & 50.0 \\
\hline $\mathrm{G}$ & 83.3 & & & 25.0 & \\
\hline $\mathrm{H}$ & 69.2 & & & 38.5 & \\
\hline Total & 69.6 & 21.5 & 21.5 & 35.2 & 12.7 \\
\hline
\end{tabular}

fue bastante menor, siendo el ooquiste de Isospora el más representativo (Fig. 1C). La Fig. 1D muestra huevos de las formas parasitarias más frecuentes encontradas en las muestras de heces (huevos tipo estrongílido y ooquistes de Isospora).

El Cuadro 2 presenta la frecuencia del tipo y forma parasitaria encontrada según la plaza pública muestreada. El total de las muestras colectadas en cuatro de las ocho plazas de Temuco fueron positivas a alguna forma parasitaria (Plaza de Armas Aníbal Pinto, Plaza Dagoberto Godoy, Feria Libre y Av. Aníbal Pinto, y Av. General Prieto Norte), en tanto que el 38\% de muestras positivas en la Plaza Recabarren (D) fue significativamente menor al resto de plazas muestreadas $(\mathrm{p}<0.05)$, la cual corresponde a uno de los sectores más céntricos. El $89.2 \pm 3.0 \%$ de las muestras fecales fueron positivas a formas parasitarias. De estas, el $19.6 \pm 3.0$ y $21.5 \pm 5.0 \%$ presentaron bi y triparasitismo, respectivamente, en tanto que $6.8 \pm 2.0 \%$ presentaron tres o más combinaciones de formas parasitarias, donde los huevos de estrongílidos fueron los más frecuentes (Cuadro 3).

\section{Discusión}

Los resultados demuestran que existe un importante número de elementos de diseminación parasitaria en parques y plazas públicas de la ciudad de Temuco; en este caso, se encontró la presencia de huevos de helmintos y ooquistes protozoarios en el $89 \%$ de las muestras (Fig. 1A). Estos resultados, aunque no del todo comparables, son mayores al $36.3 \%$ de muestras positivas a huevos de parásitos encontradas en el sector centro de la ciudad de Temuco, donde se trabajó con muestras de tierra (Armstrong et al., 2011), y levemente más altos que el $77 \%$ de contaminación parasitaria en esta ciudad en muestras de material fecal en la vía pública (Oberg et al., 2001). 
Cuadro 3. Frecuencia (\%) de poliparasitismo gastrointestinal en muestras fecales de perros en ocho plazas públicas en la ciudad de Temuco, Chile (2012)

\begin{tabular}{llc}
\hline $\begin{array}{l}\text { Asociaciones } \\
\text { parasitarias }\end{array}$ & Huevos de & $\begin{array}{c}\text { Frecuencia } \\
(\%)\end{array}$ \\
\hline Biparasitismo & Trichuris spp, estrongílidos & 5.8 \\
& Capillaria spp, estrongílidos & 4.9 \\
& Toxocara spp, estrongílidos & 2.9 \\
\multirow{2}{*}{ Triparasitismo } & Trichuris spp, Capillaria spp, estrongílidos & 7.8 \\
& Toxocara spp, Trichuris spp, estrongílidos & 5.8 \\
& Toxocara spp, estrongílidos, Strongyloides spp & 4.9 \\
\hline
\end{tabular}

La alta prevalencia en el centro de la ciudad de Temuco refleja la gran importancia del perro como diseminador de especies parasitarias, algunas de ellas de carácter zoonótico. Esto se puede deber a varios factores, entre los cuales está la elevada población de perros vagos en la ciudad: no obstante, en otras ciudades del país se han reportado frecuencias elevadas; por ejemplo, el $78 \%$ en el área rural de Folilco, en la Región de Los Ríos (Sandoval, 2003). En otros países latinoamericanos se reportan valores bastante más bajos; es así, $2.5 \%$ en Quindío, Colombia (Giraldo et al., 2005), 19.8\% en Ica, Perú (Trillo-Altamirano et al., 2003), 22.2\% en Mar del Plata, Argentina (Andresiuk et al., 2003) y 55\% en Ciudad Bolívar, Venezuela (Devera et al., 2008), en tanto que en Murcia, España, se reportó $72 \%$ de prevalencia de endoparasitismo (MartínezCarrasco et al., 2007).

En el 69\% de muestras positivas se encontraron huevos tipo estrongílidos (Fig. 1B). Este porcentaje supera al $59.5 \%$ de Uncinaria stenocephala encontrado en materia fecal recolectada en calles de Temuco (Oberg et al., 2001). Asimismo, en el estudio de Devera et al. (2008) en Ciudad Bolívar, Venezuela, la forma parasitaria más frecuente en muestras de heces fue de Ancylostoma spp (61.1\%), en tanto que en la ciudad de La
Plata, Argentina, se encontró que el $13.2 \%$ de las muestras de tierra de los parques estaban contaminadas con huevos de Toxocara spp (Fonrouge et al., 2000).

El 21\% de muestras positivas a huevos de Toxocara (Fig. 1B) puede compararse con el $12.4 \%$ de prevalencia encontrada en el año 2011 en muestras de tierra (Armstrong et al., 2011) y con el $13.3 \%$ en materia fecal en la vía pública (Oberg et al., 2001), ambos en Temuco, mientras que en un estudio en Santiago de Chile se reportó $18.2 \%$ (Salinas et al., 2001). En ciudades de otros países se ha reportado, por ejemplo, $16 \%$ de positividad para T. canis en Corrientes, Argentina, a partir de muestras de material fecal (Milano y Oscherov, 2005), de $13.2 \%$ en muestras de tierra en La Plata, Argentina (Fonrouge et al., 2000), de 28.8 y $16.7 \%$ en muestras de suelo y heces, respectivamente, en Ciudad Bolívar, Venezuela (Devera et al., 2008), y de $68.3 \%$ para Toxocara spp en La Habana, Cuba (Laird et al., 2000).

$\mathrm{Al}$ igual que otros estudios, tanto en vía pública como en casa/habitación, el poliparasitismo sigue siendo la asociación parasitaria más frecuente, alcanzando el $48 \%$ en el presente estudio (Fig. 1A), dentro del cual el triparasitismo destaca con un $21.5 \%$ (22/102, Fig. 3). Cabe destacar que en un 
estudio local a través de hallazgos de necropsia se reportó $65 \%$ para esta asociación (Oberg et al., 2001).

Los resultados del presente estudio permiten sugerir que una gran cantidad de perros, ya sean callejeros o con dueño, contaminan las áreas verdes de lugares públicos como plazas y parques. Esto refleja un desconocimiento por parte de la población de la existencia de parásitos y la escasa educación para el control y prevención de las parasitosis. Por otro lado, los resultados demuestran un problema de salud pública, ya que los canes comparten espacios comunes con las personas.

\section{Conclusiones}

Existe una alta contaminación ambiental con formas parasitarias de tipo gastrointestinal, algunas de tipo zoonótico, en plazas públicas del centro de la ciudad de Temuco, a través de las heces de perros.

\section{Agradecimiento}

Jorge Parodi recibe aportes del proyecto MECESUP UCT0804. Pamela Olivares recibe aporte del convenio de asistencia técnica UCT 278-2450.

\section{Literatura Citada}

1. Acha N, Szyfres B. 2003. Zoonosis y enfermedades transmisibles comunes al hombre y a los animales. $3^{a}$ ed. Washington DC: Organización Panamericana de la Salud. 398 p.

2. Aguirre J. 2006. Comparación de dos técnicas coprológicas para el diagnóstico de endoparasitosis del perro. Tesis de Médico Veterinario. Valdivia: Universidad Austral de Chile. 23 p.

3. Andresiuk M, Denegri G, Esardella $N$, Hollmann P. 2003. Encuesta copro- parasitológica canina realizada en plazas públicas de la ciudad de Mar del Plata, Buenos Aires. Parasitol Latinoam 58: 17 22.

4. Armstrong WA, Oberg C, Orellana JJ. 2011. Presencia de huevos de parásitos con potencial zoonótico en parques y plazas públicas de la ciudad de Temuco, Región de La Araucanía, Chile. Arch Med Vet 43: 127-134.

5. Boch J, Supperer R. 1982. Parasitología en Medicina Veterinaria. Buenos Aires: Hemisferio Sur. 55 p.

6. Devera R, Blanco Y, Hernández $H$, Simoes D. 2008. Toxocara spp. y otros helmintos en plazas y parques de Ciudad Bolívar, estado Bolívar (Venezuela). Enferm Infecc Microbiol Clin 26: 23-26.

7. Diez-Baños P, Diez-Baños $N$, Morrondo M. 1999. Nematodosis: toxocariosis, toxascaridiosis, ancilostomatidosis, tricuriosis, estrongiloidosis, espirocercosis y olulanosis. En: Cordero del Campillo M (ed). Parasitología Veterinaria. Madrid: McGraw-Hill Interamericana. p 637-651.

8. Faust C. 1938. A critical study of clinical laboratory techniques for the diagnosis of protozoan cysts and helminth eggs in faeces. Am J Trop Med Hyg 18: 169-183.

9. Fonrouge R, Guardis M, Radman N, Archelli S. 2000. Contaminación de suelos con huevos de Toxocara sp. en plazas y parques públicos de la ciudad de la Plata, Buenos Aires, Argentina. Bol Chil Parasitol 55: 83-85.

10. Giraldo M, García N, Castaño J. 2005. Prevalencia de helmintos intestinales en caninos del departamento del Quindío. Biomédica 25: 346-352.

11. Glickman T, Schanz P. 1981. Epidemiology and pathogenesis of zoonotic toxocariasis. Epidemiol Rev 3: 230-250.

12. Laird R, Carvallo D, Reyes E, García $\boldsymbol{R}$, Prieto V. 2000. Toxocara sp. en parques y zonas públicas de la Ciudad de la Habana, 1995. Rev Cubana Hig Epidemiol 38: 112-116. 
13. López J. 2006. Parásitos intestinales en caninos y felinos con cuadros digestivos en Santiago, Chile. Consideraciones en Salud Pública. Rev Med Chil 134: 193200.

14. Martínez-Carrasco C, Berriatua E, Garijo M, Martínez J, Alonso FD, Ruiz de Ybáñez R. 2007. Epidemiological study of non-systemic parasitism in dogs in southeast Mediterranean Spain assessed by coprological and postmortem examination. Zoonoses Public Hlth 54: 195-203.

15. Milano A, Oscherov E. 2005. Contaminación de aceras con enteroparásitos caninos en Corrientes, Argentina. Parasitol Latinoam 60: 82-85.

16. Noemí I, Rugiero E. 1998. Larva migrantes. En: Atías A(ed). Parasitología Médica. Madrid: Ed Mediterráneo. p 334-337.

17. Oberg C, Herrera $C$, Moreno J, Fonseca F. 2001. Parásitos de perro problema ambiental y salud pública. En: I Congreso Chileno de Bioanálisis. Iquique, Chile.

18. Salinas P, Matamala M, Schenone $\mathrm{H}$. 2001. Prevalencia de hallazgo de huevos de Toxocara canis en plazas de la
Región Metropolitana de la ciudad de Santiago, Chile. Bol Chil Parasitol 57: 3-4.

19. Salinas $P$, Reyes L, Sotomayor M, Lentoja T. 1987. Prevalencia de huevos de Toxocara sp. en algunas plazas y parques públicas de la región Metropolitana de Santiago de Chile. Bol Chil Parasitol 42: 33-36.

20. Sandoval B. 2003. Determinación coproscópica de la fauna parasitológica en perros (Canis familiaris), en el área rural de Folilco, comuna de Los Lagos, provincia de Valdivia, Décima Región, Chile. Tesis de Médico Veterinario. Valdivia: Universidad Austral de Chile. $44 \mathrm{p}$.

21. Soulsby EJL. 1965. Textbook of veterinary clínical parasitology. Oxford: Blackwell Scientific Publication. 1120 p.

22. Teuscher E. 1965. A new single method of examine faeces for diagnosis of helminth disease of rumiants. Zbl Veterinärmedizin 12: 241-249.

23. Trillo-Altamirano M, Carrasco A, Cabrera $R$. 2003. Prevalencia de helmintos enteroparásitos zoonóticos y factores asociados en Canis familiaris en una zona urbana de la ciudad de Ica, Perú. Parasitol Latinoam 58: 136-141. 